\title{
Universidad y Cooperativismo. Delimitación del Concepto de Cooperativa en una Sociedad Democrática avanzada: Referencia a los Principios Cooperativos y a su discutida vigencia
}

\author{
Dr. Enrique Gadea
}

Universidad de Deusto

Sumario: 1. Consideraciones preliminares.-2. Concepto de cooperativa: notas para una delimitación adecuada.-3. Los principios cooperativos y su discutida vigencia.-Bibliografía.

\section{Consideraciones preliminares}

En este ámbito, una de la funciones de la Universidad, utilizando las palabras del Quinto Principio Cooperativo, «es informar al público, y especialmente a los jóvenes y a los líderes de opinión, de la naturaleza y beneficios de la cooperación». Por ello, en nuestro trabajo anual vamos a intentar exponer las ideas básicas para explicar un concepto actual de cooperativa.

La definición y delimitación de la cooperativa ha supuesto siempre un trance difícil para la doctrina científica. A nuestro juicio, ello ha venido motivado fundamentalmente por dos factores principales:

1. Por la dificultad de determinar un concepto uniforme para las distintas épocas y para los distintos países.

Como es sabido, el movimiento cooperativo moderno, que debe situarse en la segunda mitad del siglo XIX, nace como reacción de las clases trabajadoras frente a los excesos del sistema capitalista propio de la época y con el objetivo de corregir primero y superar después, a través de la cooperativización de la economía y la sociedad, el sistema capitalista (LLOBREGAT, 1991, p. 4; La utopía de la cooperación tiene su más amplia representación en C. Gide y la «Escuela de Nimes»: sobre el tema, LAMBERT, 1970, p. 121 y DRIMER, A. y B., 1981, pp. 267 y ss.). 
Más tarde, las concesiones integrales que atrajeron a tan ilustres cooperativistas, ha dado paso a visiones más realistas, hasta el punto que, en la actualidad, la cooperativa, en determinados países, constituye una de las alternativas para la actuación en el marco de un sistema de economía privada.

Sin embargo, nos referimos únicamente a determinados países porque a la hora de ofrecer un concepto de cooperativa nos encontramos también con un condicionante geográfico. Es cierto que los principios cooperativos pretenden poner de relieve la coincidencia sustancial del fenómeno cooperativo con independencia del ámbito territorial donde tenga lugar (a saber: lo que caracteriza al fenómeno cooperativo es el objetivo de satisfacer necesidades a través de una empresa de propiedad conjunta y gestión democrática), pero no lo es menos que el papel que desempeñan las cooperativas dista mucho de ser coincidente: en los países menos desarrollados, prevalece la función de reacción respecto a los desequilibrios del sistema (en este ámbito, la cooperativa está destinada, en muchas ocasiones, a ser sólo un correctivo marginal de éste), mientras que, en los países de nuestro entorno, toma mayor protagonismo la orientación posibilista, que, si bien no excluye las funciones originarias, tiene muy presente el fortalecimiento y la adecuada articulación del fenómeno cooperativista, destinado no a cambiar el sistema o a sustituirlo, según ciertas concepciones de los utopistas de la cooperación económica, sino a encomendarle una función integradora en el mismo (VERRUCOLI, 1965, p. 102).

2. ${ }^{\circ}$ Por el tradicional confusionismo terminológico en una materia en la que se han mezclado ideas de la sociología y del humanitarismo junto con conceptos jurídicos y económicos.

En este apartado, vamos a realizar un análisis de lo qué es (y debe ser) la cooperativa tomando como referencia el modelo funcional o economicista alemán (sin que ello signifique no tomar en consideración las ideas de la concepción social de la legislación francesa), en el que el fenómeno cooperativo ya no constituye simplemente un instrumento de reacción de economías débiles, sino un modelo funcional de sólidos y complejos organismos cooperativos que se desarrollan libremente, sin rémoras de orden dimensional impuestas directa o indirectamente por el legislador.

Y es que, como es en la actualidad comúnmente aceptado, las posibilidades de desarrollo de la cooperación están en función de la consideración de ésta desde una perspectiva más amplia, según el tipo alemán. La Ley de cooperativas alemana no se ha fijado previamente objetivos determinados de carácter marcadamente clasista, es decir, no se ha preocupado tanto de suministrar un instrumento jurídico adecuado a las exigencias de 
ciertas economías de mercado muy débiles, como de procurar un instrumento que pudiera satisfacer también las necesidades de los sujetos de una fuerza económica media e incluso alta. Se trata de una cooperación que se ha liberado completamente de preocupaciones de principio, por lo que respecta a la estructuración en sentido capitalista de la cooperativa, dejando a los sujetos la posibilidad de utilizarla para desarrollar cualquier actividad económica licita en lugar de la sociedad anónima sobre la base de una democracia rigurosamente personal, no fundada en la entidad de la participación capitalista de cada socio. Se recurre, por tanto, al modelo cooperativo por la importancia que se quiere dar, en la organización creada para satisfacer una necesidad común de sus miembros, al principio de democracia personal (DIVAR, 1990, p. 76).

Es evidente la evolución de la función social de las cooperativas, que, si bien nacieron al socaire de un movimiento clasista, se han convertido en un instrumento técnico susceptible de ser útil a las más diversas clases sociales.

Para afrontar este reto, y que la organización cooperativa pueda ocupar un papel relevante dentro de la actividad económica, es necesario que se le proporcione un régimen legal que le permita actuar en el mercado con idénticas posibilidades que el resto de las empresas con las que debe competir. Ello exige la creación de un tipo organizativo que, en lo externo, se acerque a una organización tipo sociedad lucrativa.

Como tan acertadamente ha señalado DIVAR (1985, p. 48), estamos ante un modelo de "Cooperativismo remodelado» para que las cooperativas consigan sus objetivos en un mercado de feroces rivalidades; por ello, parece que externamente han renunciado a la puridad de sus principios $y$, singularmente, a la solidaridad y los fines sociales.

Por otra parte, para evitar confusionismo terminológico, y sin perjuicio de que adelantemos que el carácter societario de la cooperativa deriva de su legitimo derecho a una organización eficaz, haremos en este punto una referencia descriptiva a las directrices que deben tomarse en consideración para la delimitación y caracterización de la cooperativa en España, de acuerdo con las bases expuestas anteriormente, y dejaremos el análisis de los aspectos de carácter estrictamente jurídico (como la referida naturaleza societaria y la cuestión del ánimo de lucro) para otro momento.

\section{Concepto de cooperativa: notas para una delimitación adecuada}

En la actualidad, es aceptado de forma mayoritaria que la cooperativa es una empresa que tiene por objeto prioritario la promoción de 
actividades económicas y sociales de sus miembros y la satisfacción de sus necesidades con la participación activa de los mismos, observando los principios cooperativos y atendiendo a la comunidad de su entorno.

Esta definición se recoge en nuestra Ley más economicista: la LCPV (art. 1), pero es perfectamente válida para las cooperativas reguladas por la LCOOP y por las demás Leyes autonómicas, e incluso encaja dentro de lo dispuesto en el art. 1.3 del RSCE.

Para una adecuada delimitación, nos interesa destacar que la cooperativa es una empresa abierta, que puede operar con socios y con terceros, y que sus rasgos distintivos sobre las sociedades capitalistas deben analizarse desde la noción de participación (sobre la ubicación del fin mutualista, en sentido amplio, como esencia del tipo social cooperativo, puede verse PANIAGUA, 1997, p. 334. Aborda también ampliamente la cuestión, LLOBREGAT, pp. 93 y ss.).

\section{A. Sobre el carácter abierto de la cooperativa}

En España, hasta fecha relativamente reciente ha sido mayoritaria la postura (recogida en la Exposición de Motivos del CDC) que ha defendido que un presunto principio mutualista impedía a la cooperativa prestar sus servicios a los no socios (por todos, y desde un prisma jurídico, GARRIDO DE PALMA, 1981, p. 20).

En la doctrina, puede verse en este sentido, lo apuntado por ALTHAUS (1977, p. 108 y bibliografía allí citada): «...para que sea plenamente congruente con su fundamento económico, las cooperativas no deben operar, en principio, más que con sus asociados, en cuanto atañe a la prestación de los servicios que constituyen su objeto».

Sin embargo, esa postura no encaja en el planteamiento de los pioneros de Rochadle, que fue eminentemente realista.

El art. 1 de los Estatutos originarios de 1844 establece que: «La sociedad tiene por fines y como planes tomar medidas con vistas al interés pecuniario y al mejoramiento de las condiciones sociales y familiares de sus miembros...» y el art. 11, aprobado por la Asamblea General de 23 de octubre de 1854, contempla la posibilidad de venta a los no miembros, al señalar que: "Los beneficios realizados para la provisión de mercancías a personas no miembros se dedicarán a la reducción (del valor) de las mercancías en existencia» (un comentario sobre el precepto, puede verse en HOLYOAKE, 1973, p. 43).

$\mathrm{Ni}$ tampoco puede decirse que se encuentra recogida en los principios cooperativos. Solo en la documentación del XIV Congreso de la $\mathrm{ACl}$, celebrado en Londres en 1934, se prescribía como hipotético prin- 
cipio la venta exclusiva a los socios. Pero ya en el XV Congreso de París de la $\mathrm{ACl}$ de 1937, en que se realiza la primera formulación oficial de los principios, no se hace mención a ese principio entre los específicos del movimiento cooperativo, como tampoco aparece en la última versión (a la que nos referiremos en el apartado siguiente) reelaborada por la $\mathrm{ACl}$ en el Congreso XXXI, celebrado en Manchester, para conmemorar el centenario de la Alianza.

En la actualidad, debe ser aceptado que la utopía de las empresas cooperativas sólo adquiriría verdadera entidad y podría ser plenamente vinculante en un sistema económico cooperativo (VERRUCOLI, 1958, p. 101).

En una economía de mercado se consideran inviables las posturas que no admiten la realización de operaciones con terceros por considerarlas incompatibles con lo que consideran el fin principal de la entidad: la supresión del «lucro» (en sentido sociológico, de beneficios que van al capital) de la intermediación (LUCAS FERNÁNDEZ, 1973, p. 12).

Hoy no puede justificarse que la actividad de la cooperativa haya estado acotada por unos límites tan estrechos en relación con las sociedades capitalistas, y más, teniendo en cuenta que el artículo 129.2 CE, cuando se refiere a estas entidades, no impone contenidos, pautas, ni condiciones de estricta observancia al legislador ordinario.

La oposición a este planteamiento no tiene sustento jurídico ni lógico, sólo puede encuadrarse «dentro del viejo y rastrero interés de evitar molestas competencias» (DIVAR, 1985, p. 42).

Las cooperativas, al igual que las demás empresas, actúan o pueden actuar en el mercado para el cumplimiento de sus fines. En este sentido, puede decirse que no estamos ante una sociedad necesariamente mutualista (en el sentido de no poder realizar prestaciones en relación con el objeto social más que entre los socios y la sociedad) sino ante un tipo societario de configuración (interna) distinta que opera, como los demás, en beneficio de sus propios socios (VERRUCOLI, 1958, p. 104) y que, por tanto, debe extraer (o, por lo menos, deben tener la posibilidad de extraer, si así lo deciden sus socios) la máxima utilidad de las estructuras organizativas de la entidad.

Por ello, no solo es inadmisible no permitir que las cooperativas realicen operaciones con terceros (en base al principio de adhesión voluntaria y abierta, los terceros, si lo desean, tienen expedito el camino para ingresar en cualquier momento en la esfera societaria y con ello satisfacer sus intereses y necesidades como socios: VIVANTE, 1932, p. 323), sino también limitar el volumen de estas operaciones (como todavía, en la actualidad, podemos observar en la Legislación española: sobre el tema, VARGAS VASSEROT, 2006, 199 y ss.). 
En nuestro país, debido a la tradicional confusión entre Cooperativismo y Mutualismo está muy extendida la idea (hasta el punto de que se recoge a nivel legal) de que las cooperativas sólo pueden realizar el volumen mínimo de operaciones con terceros para asegurar su viabilidad económica. Este error de planteamiento en la configuración de la entidad ha sido determinante en el desarrollo del tipo y ha supuesto un obstáculo para que las cooperativas cumplan su verdadera función.

Como ha señalado ARANZADI (1976, p. 209), debe potenciarse a la cooperativa como empresa como condición ineludible para que pueda entrar en el mundo industrial, aún irredento, y tener así un quehacer propio.

El objeto de una cooperativa consiste (debe consistir) en el ejercicio en común de una actividad económica. En la realización de su objeto social, la empresa cooperativa debe actuar en base a los mismos principios económicos que cualquier otra empresa (BALLESTERO, 1983, pp. 102 y ss. y 1990, pp. 237 y ss.). Y como cualquier otra, su actuación no puede perder de vista el mercado, dado que, incluso, en aquellos casos en que la sociedad se configura, porque así lo exija la naturaleza de la actividad o la voluntad de sus miembros, para prestaciones sólo entre socios, estará directamente mediatizada por las pautas del éste ya que, sino presenta una estructura competitiva y proporciona las prestaciones que constituyen su objeto en mejores condiciones que en aquél, no podrá cumplir el fin social y, por tanto, perderá su razón de ser.

Dicho con toda claridad, no vemos ninguna dificultad para que una cooperativa, al igual que cualquier sociedad lucrativa, obtenga beneficios sociales de la intermediación (repárese en que el intermediario no se elimina, sólo se sustituye: PANIAGUA, 1997, p. 326). La diferencia debe residir en la forma de distribución (BALLESTERO, 1990, p. 237): en el caso de las sociedades lucrativas, la distribución a los socios se realizará en función del capital aportado y en el caso de las cooperativas, después de atender los fondos obligatorios (incluido el FEP), la adjudicación a sus miembros se realizará en proporción a las operaciones, servicios o actividades realizadas por cada uno en la entidad.

B. La participación como elemento distintivo de la cooperativa frente a las sociedades capitalistas

Para conseguir la eficiencia del sistema cooperativo es necesario que las propias cooperativas constituyan auténticas estructuras democráticas participativas en la que sus miembros vean satisfechas sus necesidades y reflejadas sus visiones. 
La especial estructura participativa de las cooperativas se vertebra en torno a tres pilares:

1. El papel del socio: en la cooperativa, los socios participan no sólo aportando las sumas de capital necesarias sino operando como clientes, en las cooperativas de consumo, y como trabajadores, en las cooperativas de producción. Es indudable que esa doble posición de sus miembros constituye una característica propia (aunque no exclusiva: se da también, por ejemplo, en las mutuas de seguros o en las sociedades de garantía reciproca) de las cooperativas, que tiene trascendencia en el plano legal $y$, especialmente, en la configuración del estatus jurídico del socio, al que, por ejemplo, se le reconoce el derecho y se le impone el deber de participar en la actividad cooperativa.

2. La gestión democrática por parte de sus miembros, basada en la asociación igualitaria, que, en la práctica, se concreta, por lo menos en las cooperativas de primer grado, en la regla general de que los socios tengan iguales derechos de voto (un socio, un voto).

3. El sistema de distribución de beneficios, al margen de la contribución en el capital, en proporción a las operaciones, servicios o actividades realizadas por cada socio en la entidad.

A nuestro juicio, el hecho que justifica la especificidad de la sociedad cooperativa no es la gestión preferentemente mutualistica: el elemento definitorio de la cooperativa es que constituye una iniciativa empresarial por parte de un grupo de personas que, al margen de la participación capitalista de cada socio, tiene por finalidad la satisfacción de sus necesidades mediante la recuperación de la forma de empresa personalista y de funcionamiento interno democrático. Ese es el hecho diferencial sobre el que debe basarse la particular consideración y el adecuado tratamiento (incluso en el plano fiscal) para la entidad asociativa cooperativa.

Como se desprende del artículo 129.2 de nuestra Constitución, la cooperativa se presenta como una fórmula jurídica adecuada para facilitar el acceso de los trabajadores a la propiedad de los medidos de producción y para canalizar las formas participativas en las empresas.

El artículo 129.2 de la CE establece que: «los poderes públicos promoverán eficazmente las diversas formas de participación en la empresa y fomentarán, mediante una Legislación adecuada, las sociedades cooperativas». Este precepto constituye un mandato dirigido a los poderes públicos para que éstos fomenten las sociedades cooperativas, es decir, deben tomar las medidas adecuadas para que el modelo eco- 
nómico constitucional de economía social de mercado se nutra de empresarios cooperativos. Con ello, nuestra Constitución de 1978 sitúa a la sociedad cooperativa, adecuándose para ello a las exigencias actuales y recogiendo las tendencias más modernas de las Legislaciones europeas sobre cooperación, en el contexto adecuado de la participación, contrastando así con otras Constituciones, como la italiana de 1947, que en su artículo 45 adopta la postura clásica, más restrictiva y parcialmente superada en relación con el Cooperativismo, al señalar que: "La República reconoce la función social de la cooperación con carácter de mutualidad y sin fines de especulación privada ...» (en Italia, las formulaciones doctrinales que se posicionan a favor o en contra de que la esencia de la cooperativa descanse o no sobre el fin mutualista son muy numerosas; a favor: se han pronunciado ASCARELLI, OPPO o BOTTERI; en contra, FERRI O VERRUCOLI. Una amplia referencia a la cuestión, puede verse en PANIAGUA, 1997, p. 338 y ss.).

El enfoque de la Constitución tiene una especial importancia, desde un punto de vista general, en cuanto que en una "sociedad democrática avanzada», utilizando las palabras del Preámbulo de la Constitución, la participación laboral tiene, como señala DIVAR, (1990, p. 87), un basamento como valor social reivindicable, y en ello consisten a efectos prácticos la justicia social y la ética social, sustento del Derecho justo. La participación, en su esencia y últimas consecuencias, supone «el reparto del poder». Por ello la participación auténtica en sentido absoluto es la derivada de la consideración como socio del trabajador (cogestión), la que establece dándole al contrato de trabajo una vis societaria como, precisamente, realiza el Cooperativismo.

Por tanto, lo relevante es que la cooperativa constituye el modelo de empresa participativa, que basa su funcionamiento interno en principios de verdadera democracia económica (DIVAR, 1990, 87 y ss.).

Sobre la base de esa consideración, exigimos un modelo de cooperativa, ajena a las restricciones del pasado, abierto, atractivo (desde el punto de vista de sus utilidades) y acorde con los nuevos tiempos. Estamos ante una fórmula jurídica para la organización económica, sujeta a los principios y fines que le son específicos, que tiene (debe tener) la misma extensión técnica y económica que una sociedad capitalista (anónima o limitada) (DIVAR, 1985, p. 43). De lo contrario, el modelo cooperativo quedará condenado a la condición de marginal, en beneficio de las opciones verdaderamente capitalistas. Este planteamiento es, además, coherente con el principio constitucional de libertad de empresa recogido en el artículo 38 de la C.E. que, en lo que concierne a este punto, comporta el derecho a que los ciudadanos adopten la organización empresarial que deseen. 


\section{Los principios cooperativos y su discutida vigencia}

Los principios cooperativos, formulados y revisados por la $\mathrm{ACl}$ cada cierto tiempo, constituyen pautas flexibles mediante las que se delimita la naturaleza democrática de las cooperativas, el papel de los diferentes participes y la forma de distribuir los excedentes creados. Los principios determinan las cualidades esenciales que hacen que las cooperativas sean diferentes a los otros tipos de empresa y que el movimiento cooperativo sea valioso. Por tanto, su importancia dogmática es evidente, aunque su trascendencia jurídica queda supeditada a los términos en que hayan sido incorporados en las respectivas legislaciones internas, lo que condiciona notablemente su posible eficacia jurídica como criterios correctores de las posibles «impurezas» del legislador correspondiente. Ello, no obstante, su carácter informador de la estructura y funcionamiento de la cooperativa les adiciona un claro valor como elemento de interpretación de la normativa cooperativa (MARTíNEZ SEGOVIA, 2001, pp. 62 y 63, PANIAGUA, 2005, p. 37).

Como ha quedado señalado, la versión actual de los principios cooperativos (las dos anteriores se aprobaron en los Congresos de Paris de 1937 y en el de Viena de 1966) se contiene en la «Declaración de la Alianza Cooperativa Internacional sobre Identidad Cooperativa», adoptada en el Congreso celebrado en Manchester en 1995 para conmemorar el centenario de la Alianza.

Entre 1990 y 1992, bajo la dirección de Sven Böök, miembros de la $\mathrm{ACl}$ e investigadores independientes analizaron, mediante una extensa discusión, la naturaleza de los valores cooperativos. Los resultados de ese estudio están recogidos en el libro: «Los Valores Cooperativos en un mundo en cambio», escrito por Böök y publicado por la $\mathrm{ACl}$. Este libro, junto con «Principios Cooperativos: Hoy y mañana», escrito por W. P. Watkins, constituyen los pilares sobre los que se asienta la Declaración sobre Identidad Cooperativa.

Sobre los valores cooperativos, la Declaración de la Alianza señala que las cooperativas están basadas en los valores de la autoayuda, la autorresponsabilidad, la democracia, la igualdad, la equidad y la solidaridad. Además, destaca que, siguiendo la tradición de sus fundadores, los socios cooperativos - sin descartar que puedan encontrarse en otro tipo de organizaciones - hacen suyos los valores éticos de la honestidad, la transparencia, la responsabilidad y la vocación sociales.

En ella, se enumeran siete principios: Adhesión Voluntaria y Abierta; Gestión Democrática por parte de los socios; Participación Económica por parte de los socios; Autonomía e Independencia; Educación, Formación e Información; Cooperación entre Cooperativas; e Interés por 
la Comunidad. Los tres primeros principios se dirigen a la dinámica interna típica de cualquier cooperativa. Los cuatros últimos se dirigen tanto al funcionamiento interno como a las relaciones externas de las cooperativas.

En la Declaración de 1995 los principios han sido formulados de la forma siguiente:

1. Adhesión Voluntaria y Abierta: «Las cooperativas son organizaciones voluntarias, abiertas a todas las personas capaces de utilizar sus servicios y dispuestas a aceptar las responsabilidades de ser socio, sin discriminación social, política, religiosa, racial o de sexo».

Del primer principio merece especial atención la frase que afirma que las cooperativas están "abiertas a todas las personas capaces de utilizar sus servicios y dispuestas a aceptar las responsabilidades de ser socio, sin discriminación, política, religiosa, racial o de sexo». Esta declaración reafirma el compromiso de las cooperativas con el reconocimiento de la dignidad fundamental de todos los individuos.

2. 'Gestión Democrática por parte de los socios: "Las cooperativas son organizaciones gestionadas democráticamente por los socios, los cuales participan activamente en la fijación de sus políticas y en la toma de decisiones. Los hombres y las mujeres elegidos para representar y gestionar las cooperativas son responsables ante los socios. En las cooperativas de primer grado, los socios tienen iguales derechos de voto (un socio, un voto) y las cooperativas de otros grados están también organizadas de forma democrática».

La regla para las cooperativas de primer grado es clara; en cambio, plantea más dudas la interpretación del último inciso. En el Informe de la Alianza (que explica las razones que han llevado a la adopción de la Declaración sobre Identidad Cooperativa) se aclara que la regla para las votaciones en otros grados es abierta. Se considera que los propios movimientos cooperativos son los más capaces de definir lo que es democrático en una circunstancia dada. Incluso se reconoce que en muchas cooperativas de segundo y tercer grado, los sistemas de votación proporcional han sido adoptados para reflejar la diversidad de intereses, el número de socios en cooperativas asociadas, y el compromiso entre las cooperativas involucradas. Ahora bien, también se señala que tales acuerdos deben revisados periódicamente y que es normalmente insatisfactorio si las cooperativas más pequeñas en tales situaciones tienen tan poca influencia que prácticamente se sienten privadas del derecho de voto.

3. Participación Económica de los socios: "Los socios contribuyen equitativamente al capital de sus cooperativas y lo gestionan de forma democrática. Por lo menos parte de ese capital debe ser propiedad co- 
mún de la cooperativa. Normalmente, los socios reciben una compensación, si la hay, limitada sobre el capital entregado para adquirir la condición de socio. Los socios asignan los excedentes para todos o algunos de los siguientes fines: el desarrollo de su cooperativa, posiblemente mediante el establecimiento de reservas, parte de las cuales por lo menos serían indivisibles; el beneficio de los socios en proporción a sus operaciones con la cooperativa; y el apoyo de otras actividades aprobadas por los socios».

Este principio describe tanto cómo participan los socios en el capital, como la forma en que deben distribuirse los excedentes. Es digna de ser resaltada la frase que señala que «los socios contribuyen equitativamente al capital de las cooperativas y lo gestionan de forma democrática», dado que, por una parte, hace hincapié en la necesidad de que los socios aporten capital a su cooperativa y, por otra, permite que las diferentes legislaciones exijan que cada socio aporte, sin que ello tenga trascendencia en el proceso de adopción de decisiones, cantidades distintas, según su capacidad económica. En la asignación de excedentes resulta sorprendente la flexibilidad: "Los socios asignan los excedentes para todos o alguno de los siguientes fines ...». Sin embargo, en un sistema legal como el nuestro en el que se parte del carácter societario de la cooperativa, resulta impensable que las distintas normas no exijan la constitución de un fondo de reserva obligatorio.

4. ${ }^{\circ}$ Autonomía e Independencia: «Las cooperativas son organizaciones autónomas de autoayuda, gestionadas por sus socios. Si firman acuerdos con otras organizaciones, incluidos los gobiernos, o si consiguen capital de fuentes externas, lo hacen en términos que aseguren el control democrático por parte de sus socios y mantengan su autonomía cooperativa».

Este principio no se recogió explícitamente en la formulación de 1996. En ese momento, la $\mathrm{ACl}$ adoptó una posición más condescendiente para evitar la salida de la entidad internacional de los países de regímenes comunistas, dado que limitaban la autonomía e independencia de sus cooperativas. Ahora, tras la caída de tales regímenes, la $\mathrm{ACl}$ vuelve hacia la posición de 1937, época en la que consagró como uno de los principios la «neutralidad política y religiosa». Sin embargo, lo más novedoso en la formulación de este principio es la referencia a la firma de acuerdos «con otras organizaciones». Con ella se reconoce el hecho de que, en todo el mundo, cada vez más cooperativas están entrando en proyectos conjuntos con otras empresas del sector privado, aunque se señala la importancia de que éstas mantengan la libertad de controlar su propio destino futuro al negociar tales acuerdos. 
5. ${ }^{\circ}$ Educación, Formación e Información: «Las cooperativas proporcionan educación y formación a los socios, a los representantes elegidos, a los directivos y a los empleados para que puedan contribuir de forma eficaz al desarrollo de sus cooperativas. Informan al gran público, especialmente a los jóvenes y a los lideres de opinión, de la naturaleza y beneficios de la cooperación».

El movimiento cooperativo tiene un compromiso claro y antiguo con la educación de los socios. En este sentido, la Declaración de 1995 señala que los fondos cooperativos destinados a este fin deben dedicarse a la educación — dirigida a la comprensión de la complejidad y riqueza del pensamiento y acción cooperativos - y a la formación - dirigida a la capacitación profesional— de todos lo que están implicados en las cooperativas, no sólo de los socios, y a la información al gran público - especialmente a los jóvenes y a los lideres de opinión- de la naturaleza y beneficios de la cooperación. Se realiza especial hincapié en esta última actividad, descuidada en las últimas décadas por muchas cooperativas, que tiene gran importancia para su desarrollo futuro, ya que nadie aprecia ni apoya lo que no entiende (sobre la utilidad de la labor de los Centros de Estudios Cooperativos, puede verse lo apuntado por BENAVIDES, 1987, pp. 138 y ss.).

6. ${ }^{\circ}$ Cooperación entre cooperativas: «Las cooperativas sirven a sus socios lo más eficazmente posible y fortalecen el movimiento cooperativo trabajando conjuntamente mediante estructuras locales, nacionales, regionales, e internacionales».

Para conseguir esos objetivos - servir eficazmente a sus socios y fortalecer el movimiento cooperativo- es necesario crear estructuras. Por ello surgen las asociaciones, federaciones, confederaciones y entes interregionales e internacionales cuya cúspide es la ACI.

7. ${ }^{\circ}$ Interés por la Comunidad: «Las cooperativas trabajan para conseguir el desarrollo sostenible de sus comunidades mediante políticas aprobadas por sus socios».

En 1966, al enunciar el sexto principio de cooperación entre cooperativas, se señaló como objetivo "servir mejor a los intereses de sus miembros y de la comunidad». En 1995 se concede mayor importancia a este objetivo, atribuyendo a la «preocupación por la comunidad» el carácter de principio. Sin duda, se reconoce que las cooperativas son organizaciones que existen principalmente para el beneficio de sus socios, aunque dada la vinculación de las cooperativas a sus comunidades - a diferencia de las sociedades capitalistas que pueden llegar o irse según las condiciones coyunturales que se presenten para su desarrollolos socios no pueden obviar el fuerte compromiso social de éstas, que, independientemente de las condiciones coyunturales, deben dirigir su 
labor hacia el aumento del bienestar social, haciendo de la proyección y la acción hacia la comunidad, característica cooperativa.

Los principios son mandamientos que deben ser seguidos por las cooperativas. No obstante, como acertadamente se destaca en el Informe de la Alianza (cuyo texto en castellano puede verse, al igual que el relativo a los principios, en el Anuario de Estudios Cooperativos de la UD, pp. 73 y ss.), no es suficiente preguntar si una cooperativa está siguiendo al pie de la letra un principio, es igualmente importante saber si está siguiendo su espíritu. Si la visión que proporciona cada principio, individual y colectivamente, está arraigada en las actividades diarias de la cooperativa. Además, no pueden considerarse independientes: los principios están unidos sutilmente. Cuando se pasa uno por alto, todos se resienten. Aunque, a nuestro juicio, la vigencia efectiva del principio de gestión democracia y participativa - que no es otra cosa que la involucración de los socios en la toma de decisiones de la entidad - constituye el elemento clave para el éxito del movimiento (MASAYUKI YAMAGISHI, 1995, p. ii), es cierto que no hay que evaluar las cooperativas exclusivamente sobre la base de un principio dado, más bien, deben ser evaluadas sobre la base de cómo se adhieren a los principios en su totalidad.

\section{Bibliografía}

Althaus, Tratado de Derecho cooperativo, 2. a ed., Zeus, Rosario, 1977.

ARANZADI, Cooperativismo industrial como sistema, empresa y experiencia, Universidad de Deusto, Bilbao, 1976.

BAlleSTERo, Teoría económica de las cooperativas, Alianza Universidad, Madrid, 1983.

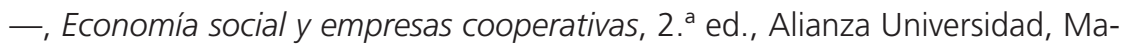
drid, 1990.

Benavides, Evolución del Pensamiento Cooperativo, Buenos Aires, 1987.

DIVAR, La alternativa Cooperativa, Ceac, Barcelona, 1985.

—, La Democracia económica, Universidad de Deusto, Bilbao, 1990.

Drimer A. y B., Las Cooperativas. Fundamento, historia y doctrina, Buenos Aires, 1981.

GARRIDO DE PALMA, «La cooperativa en el Derecho español a través de sus principios», Rev. Der. Not., 1981.

HolyOAKe, Historia de los Pioneros de Rochdale, Zaragoza, 1973.

LAMBERT, La Doctrina cooperativa, Intercoop, Buenos Aires, 1970.

LLoBREGAT, Mutualidad y empresas y cooperativas, Bosch, Barcelona, 1991.

LUCAS FERNÁNDEZ, "Nacimiento jurídico de la cooperativa», en Tribuna Cooperativa, núm, 14, 1973.

MASAYUKI YAMAGISHI, La democracia participativa en las cooperativas: cómo dar significado a la participación, Buenos Aires, 1995. 
Martínez Segovia, «Sobre el concepto jurídico de cooperativa», en Moyano Fuentes (Coord.), La sociedad cooperativa: un análisis de sus características societarias y empresariales, Universidad de Jaén, Jaén, 2001.

Paniagua, Mutualidad y lucro en la sociedad cooperativa, McGrawHill, Madrid, 1997.

- La sociedad cooperativa. Las sociedades mutuas de seguros y las mutualidades de previsión social, Vol. 1, Tomo XXII del Tratado de Derecho mercantil, Coord. por Jiménez Sánchez, Marcial Pons, Madrid, 2005.

VARGAS VASSEROT, La actividad cooperativizada y las relaciones de la cooperativa con sus socios y con terceros, Thomson-Aranzadi, Pamplona, 2006.

Verrucolı, La Societá Cooperativa, Giuffré, Milano, 1958.

- " «Tendencias sobre Derecho comparado Europeo sobre cooperación», en Anales de Moral social y económica, Centro de Estudios de la Santa Cruz del Valle de los Caídos, Vol. 9, 1965.

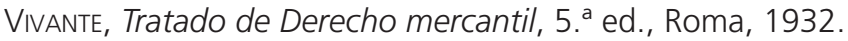

